\title{
Towards bioinspired superhydrophobic poly(L-lactic acid) surfaces using phase inversion-based methods
}

\author{
Jun Shi, Natália M Alves and João F Mano \\ 3B's Research Group, Biomaterials, Biodegradables and Biomimetics, Department of Polymer \\ Engineering, University of Minho, Campus de Gualtar, 4710-057 Braga, Portugal \\ and IBB, Institute for Biotechnology and Bioengineering, Braga, Portugal \\ E-mail: jmano@dep.uminho.pt
}

Received 28 January 2008

Accepted for publication 23 June 2008

Published 15 July 2008

Online at stacks.iop.org/BB/3/034003

\begin{abstract}
The water repellency and self-cleaning ability of many biological surfaces has inspired many fundamental and practical studies related to the development of synthetic superhydrophobic surfaces. However, the investigation of such substrates made of biodegradable polymers has been scarce. Simple approaches based on a single step, performed at room temperature (and pressure), were implemented to obtain superhydrophobic poly(L-lactic acid) (PLLA) surfaces via phase inversion-based methods, without addition of low-surface-energy compounds. Water contact angles above $150^{\circ}$ were obtained using some processing conditions. In such cases scanning electronic microscopy micrographs of such surfaces revealed a clear rough texture composed by leafy clusters with micro-nano binary structures. Such materials could be used in specific environmental and biomedical applications, namely in implantable materials or in antibacterial or antithrombogenic surfaces.
\end{abstract}

\section{Introduction}

Superhydrophobic surfaces exhibiting a water contact angle (CA) higher than $150^{\circ}$ have attracted much attention for both industrial and biological applications [1-5], such as antifogging and self-cleaning coatings for windows, microfluidics, domestic commodities or in the biomaterials area [6]. Many plants and insects exhibit surfaces that are self-cleaning. The superhydrophobicity of lotus leaves is a classical example where water drops landing on a leaf are almost spherical and may roll off, effectively enabling the elimination of dirt and particles [7]. The lotus effect is caused by the unique microscopic roughness produced by the hydrophobic epicuticular wax crystalloids. This phenomenon may be observed in many other leaves and in animals, such as the wings of butterflies and cicadae or the legs of the water strider, helping this insect to stand on the water's surface [2, 8].
Conventionally, synthetic bioinspired superhydrophobic surfaces have been produced mainly in two ways. One is to create a rough structure on a hydrophobic surface, and the other is to modify a rough surface using materials with lowsurface free energy [9]. Until now, many methods have been reported for manufacturing superhydrophobic surfaces, such as chemical vapour deposition, sacrificial colloidal particles, crystallization control, electrochemical deposition, chemical modification, etching/lithography, plasma modification and aligned carbon nanotube or polymer nanofibers [9-14]. However, simple, economical and one-step procedures to produce superhydrophobic surfaces need to be developed. For example, Erbil et al reported a simple method for forming superhydrophobic surfaces in polypropylene by combining solvents and adequate drying temperatures [15]. To our knowledge, such kinds of technologies were never implemented in biodegradable polymers. 


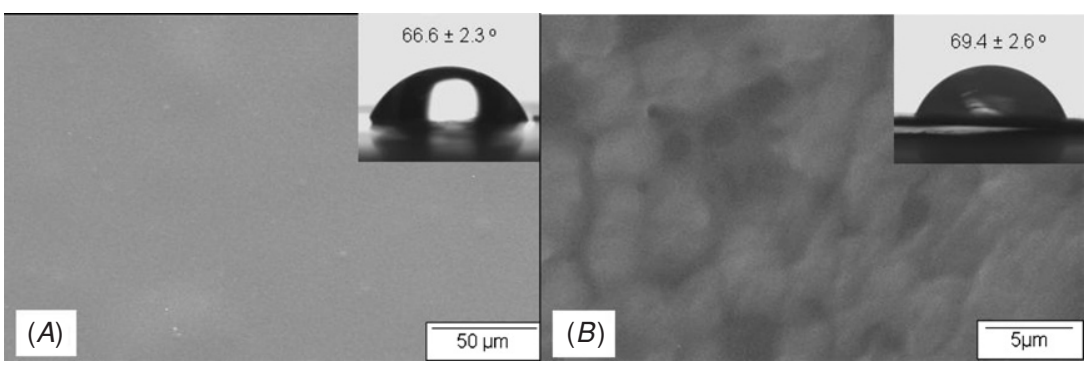

Figure 1. SEM images of the smooth surface of a PLLA film obtained by a solvent casting method $(A)$ and surface of a PLLA film obtained by precipitation in water $(B)$. The insets show photographs of a water droplet on the corresponding surfaces.

Poly(L-lactic acid) (PLLA), a biocompatible and biodegradable polyester, is one of the few synthetic degradable polymers approved by the Food and Drug Administration for a variety of human clinical and ecological applications [16-18]. In this communication, we report several methodologies based on phase inversion towards the production of superhydrophobic PLLA surfaces. Only a small number of papers concerning superhydrophobic non-biodegradable biocompatible polymers have been published, aimed at improving blood compatibility [19, 20]. Moreover, little work has been reported on superhydrophobic biodegradable polymers, such as PLLA. An example in this context is polycaprolactone membranes prepared by electrospinning followed by chemical vapour deposition [21], or by transcribing the microscopic pattern of the lotus leaf [22]. Such systems could have potential use in biomedical or environmental applications where biocompatibility and biodegradability are required. Examples include temporary microfluidics devices for tissue engineering strategies, surfaces with improved antibacterial properties or implants with enhanced blood compatibility. Note also that water repellency can also prevent hydrolysis of the polyesters, enabling one to control/retard the degradation profile of such materials [22].

In this work novel rough surfaces made of PLLA were prepared using methods that combine the use of a solvent (dioxane) and non-solvents (water or ethanol). The objective is to find the most adequate method that maximizes the hydrophobic character of the films. Moreover, we intend to find a simple method that could enable the production of large superhydrophobic surface areas.

\section{Experimental details}

Poly(L-lactic acid) (PLLA) pellets, with $M_{n}=69000$ and $M_{w} / M_{n}=1.734$, were dissolved in a fixed volume of dioxane in a test flask at room temperature. In some experiments a small amount of a non-solvent was added to dioxane, in this case $7 \%$ of water. Polymer concentrations of 5\%, 7.5\%, $10 \%$ and $12.5 \%(\mathrm{w} / \mathrm{v})$ were applied. The solutions were spread uniformly onto clean Petri dishes. Control flat membranes were produced by simple solvent casting. The resulting membranes were air dried overnight followed by vacuum drying for $48 \mathrm{~h}$ at $40{ }^{\circ} \mathrm{C}$. Different protocols to produce rough PLLA films were followed, some of them based on the procedures used to produce highly hydrophobic surfaces from poly(vinylidene fluoride) [23] or porous membranes of PLLA [24].

In a first procedure, based on an immersion-precipitation method, the PLLA/dioxane solution-cast film was immersed in water at room temperature. The obtained wet membrane was washed with water/ethanol to remove the residual dioxane. In the second method a softer coagulation bath was used, based on the work of Peng et al [23], where the solution-cast film previously conditioned at $-20{ }^{\circ} \mathrm{C}$ for $60 \mathrm{~min}$ was immersed in a mixture of dioxane/water $(65 / 35, \mathrm{v} / \mathrm{v})$ for $30 \mathrm{~min}$. In this case one expects that the precipitation and mass transfer of the solvent will be slower, resulting in membranes with a different topography and less asymmetry throughout the thickness. In a third procedure, the PLLA solution was placed in air until gelation took place, which was detected by a strong increase in viscosity. Typically $5 \mathrm{~min}$ were enough to achieve this state; then the film was immersed in ethanol at room temperature for $30 \mathrm{~min}$. In all methods employed the resulting films were dried at $40{ }^{\circ} \mathrm{C}$ for $48 \mathrm{~h}$ in vacuum. The wettability of the films was measured on a DataPhysics OCA 20 contact angle system at ambient temperature using the sessile drop method. The surface morphology of the porous films was analysed by scanning electron microscopy (SEM) using a Leica Cambridge S360 equipment. In this case, the films were previously coated with gold.

\section{Results and discussion}

The film obtained by solvent casting was found to be transparent and the SEM photograph of its surface reveals that a smooth and dense PLLA membrane could be obtained by this method-see figure $1(A)$. The inset picture presents the profile of a water droplet on this surface, exhibiting a contact angle of $66.6 \pm 2.3^{\circ}$. The film obtained by precipitating the PLLA solution in water was found to be white and opaque, but the contact angle is $69.4 \pm 2.6^{\circ}$, although some texture at the micro-scale level could be observed on its surface-see figure $1(B)$.

Additionally, we prepared the PLLA films by precipitating the polymeric solution into the mixture of dioxane/water $(65 / 35, v / v)$ or ethanol. The development of the membranes using such phase inversion methods involves complex mass transfer of the solvent, liquid-solid demixing and 

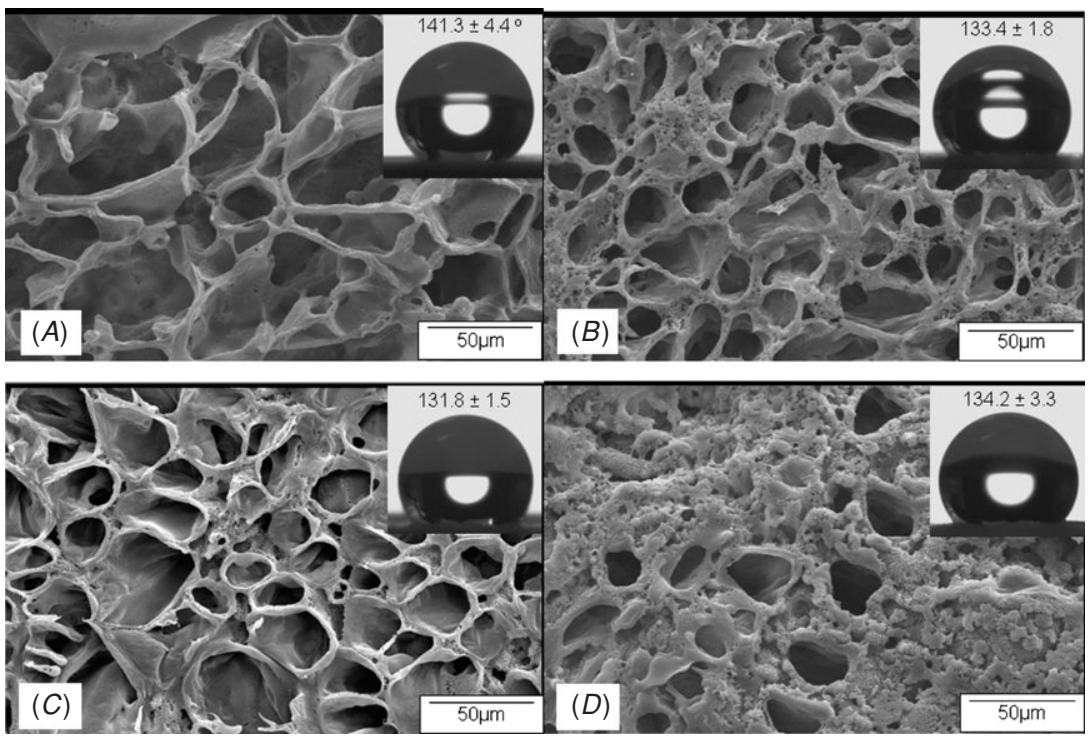

Figure 2. SEM images of the top surface of films obtained by precipitating PLLA/dioxane solution in a mixture of dioxane/water $(65 / 35, \mathrm{v} / \mathrm{v})$. Different PLLA concentrations were used: $(A)$ 5\%; $(B) 7.5 \% ;(C)$ 10\%; $(D)$ 12.5\%. Insets show photographs of a water droplet on the corresponding surfaces.

Table 1. Contact angle of PLLA films obtained by precipitation in the mixture of dioxane/water (65/35).

\begin{tabular}{lllllllll}
\hline & \multicolumn{6}{c}{ Contact angle (deg) of films prepared with different concentrations of PLLA/dioxane solution (\%, wt/v) } \\
\cline { 2 - 9 } $\begin{array}{l}\text { Position on } \\
\text { the sample }\end{array}$ & 5 & 7.5 & 10 & 12.5 & $\begin{array}{l}5(+7 \% \\
\text { water) }\end{array}$ & $\begin{array}{l}\text { w.5 }(+7 \% \\
\text { water })\end{array}$ & $\begin{array}{l}10(+7 \% \\
\text { water })\end{array}$ & $\begin{array}{l}12.5(+7 \% \\
\text { water })\end{array}$ \\
\hline Top side & $141.3 \pm 2.8$ & $133.4 \pm 2.7$ & $131.8 \pm 1.5$ & $134.2 \pm 3.3$ & $115.8 \pm 4.7$ & $126.6 \pm 2.1$ & $119.3 \pm 1.4$ & $113.8 \pm 3.9$ \\
Bottom side & $122.5 \pm 1.6$ & $119.2 \pm 2.7$ & $106.9 \pm 3.3$ & $112.9 \pm 0.8$ & $125.9 \pm 1.9$ & $122 \pm 0.5$ & $120 \pm 2.6$ & $122.1 \pm 2.9$ \\
\hline
\end{tabular}

crystallization of PLLA. As shown before in poly(vinylidene fluoride) membranes, such processes could be affected by adding some quantity of solvent in the coagulation bath, where films with different topography and water contact angles could be produced [23]. Figure 2 shows that the presence of dioxane in the coagulation bath has a strong effect on the surface topography of the obtained films, as compared with the use of pure water (figure $1(B)$ ).

The use of a softer-precipitation bath will slow down the formation of the membrane upon immersion of the solutioncast film, originating a porous structure. It is clear that the pore morphology is highly dependent on the concentration of PLLA in the dioxane solution. For lower concentrations the surface is much more porous, displaying bigger pores. With the increase of PLLA concentration a densification of the structure is observed, and in the films prepared with a $12.5 \%$ PLLA solution the number of pores is considerably lower than for the one prepared with more diluted solutions (figure 2(D)). The CAs (see insets in figure 2) demonstrate that more hydrophobic surfaces can be obtained using more diluted PLLA solutions: values of $141.3^{\circ}$ could be obtained using a 5\% PLLA solution (figure $2(A)$ ). The CA was also measured on the bottom side of the membranes-see table 1 . It is clear that the wettability can be very different on the two sides, especially for the film prepared with the more diluted solution of PLLA. The results obtained indicate that it was not possible to obtain superhydrophobic PLLA structures using this procedure, although the contact angle could be enhanced by adding dioxane in the precipitation media.

Further studies were performed using dioxane solutions of PLLA containing a small amount of a non-solvent (7\% of water) to process rough surfaces. The introduction of a nonsolvent could influence the film formation upon precipitation in the coagulation bath and a few studies showed this effect in the specific preparation of superhydrophobic polymeric films $[15,25]$. Erbil et al reported that non-solvents act as a polymer precipitator, increasing the extent of polymer phase separation between the liquid and polymer rich phases, and this also increases the crystallization rate [15]. The surface morphology of such films prepared with different PLLA concentrations is shown in figure 3. Compared with the SEM pictures of figure 2 we can conclude that the addition of water in the PLLA solution leads to films with less porous surfaces. This was reflected in a generalized decrease in the water $\mathrm{CA}$ - see insets in figure 3 and table 1 . One can therefore conclude that, in this particular case, the addition of a non-solvent in the PLLA solution had a detrimental effect in the production of hydrophobic substrates. In the final procedure used in this work just dioxane was used to prepare the PLLA solutions.

Finally, PLLA films were obtained by a gelation-in-air procedure followed by precipitation in ethanol. All films prepared by this method have a water contact angle higher than $150^{\circ}$, indicating that superhydrophobic films could be obtained-see insets in figure 4 and table 2. Although the 

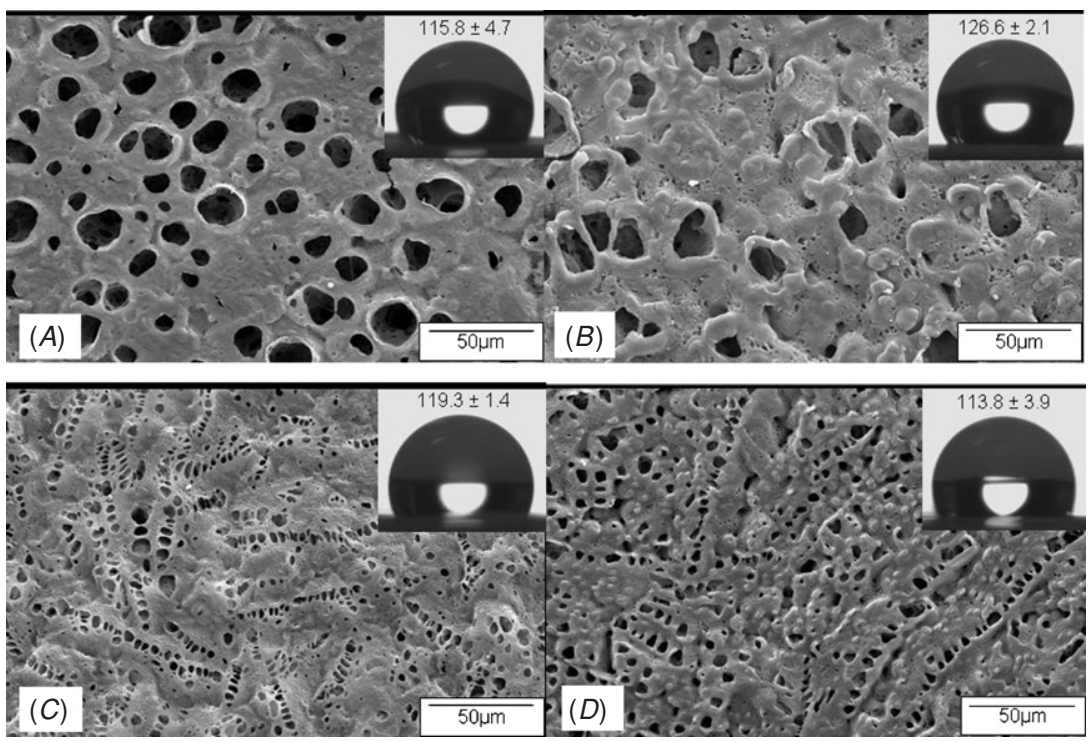

Figure 3. SEM images of the top surface of films obtained by precipitating PLLA solution in dioxane/water $(93 / 7 \mathrm{v} / \mathrm{v})$ in the mixture of dioxane/water $(65 / 35, \mathrm{v} / \mathrm{v}) .7 \%$ of water was added in PLLA/dioxane solution. Different PLLA concentrations were used: $(A) 5 \%$; (B) $7.5 \%$; (C) $10 \%$; (D) $12.5 \%$. Insets show photographs of water droplets on the corresponding surfaces.
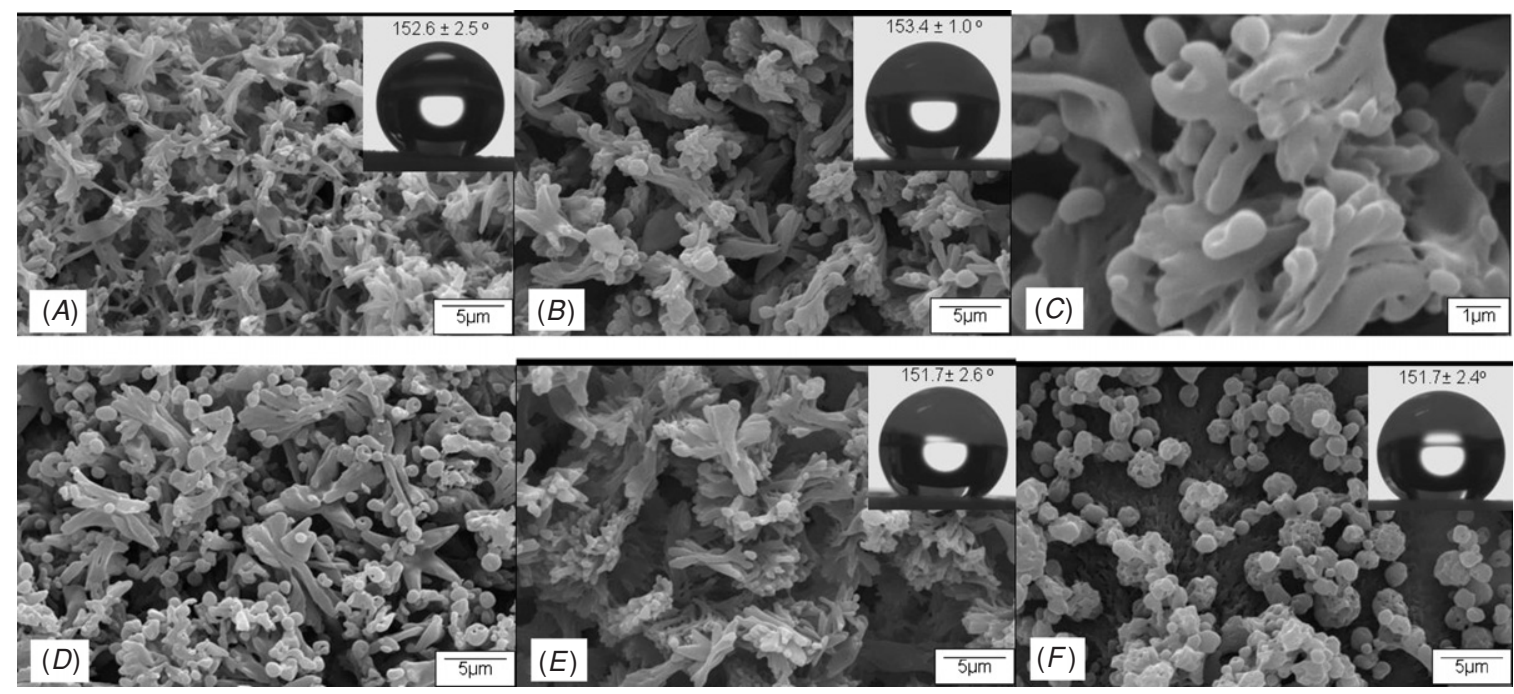

Figure 4. SEM images of the top surface of films obtained by precipitating PLLA/dioxane solutions in ethanol. Different PLLA concentrations were used: $(A) 5 \% ;(B) 7.5 \%(C$ is a magnification of sample $B, D$ is a cross section of sample $B) ;(E) 10 \% ;(F) 12.5 \%$. Insets show photographs of water droplets on the corresponding surfaces.

Table 2. Contact angle of PLLA films obtained by precipitation in ethanol.

\begin{tabular}{lllllll}
\hline & \multicolumn{5}{c}{ Contact angle (deg) of films prepared with different concentrations of } \\
PLLA/dioxane solution $(\%, w t / v)$ \\
\cline { 2 - 7 } $\begin{array}{l}\text { Position on } \\
\text { the sample }\end{array}$ & 5 & 7.5 & 10 & 12.5 & $\begin{array}{l}7.5(+7 \% \\
\text { water })\end{array}$ & $\begin{array}{l}10(+7 \% \\
\text { water })\end{array}$ \\
\hline Top side & $152.6 \pm 2.5$ & $153.4 \pm 1.0$ & $151.7 \pm 2.6$ & $151.7 \pm 2.4$ & $132.3 \pm 4.8$ & $131.2 \pm 3.2$ \\
Bottom side & $125.5 \pm 1.8$ & $129.9 \pm 3.1$ & $127.3 \pm 2.8$ & $137.5 \pm 2.1$ & $130.5 \pm 1.4$ & $136.8 \pm 2.1$ \\
\hline
\end{tabular}

contact angles were found be higher than $150^{\circ}$, and thus the surface could be defined as superhydrophobic taking into account the common nomenclature [26, 27], one must be aware that surfaces with contact angles higher than $160^{\circ}$ have also been widely reported in the literature, thus exhibiting an even higher water repellency. In order to increase the contact angle in the films developed here, surface modification could be performed, through the immobilization of hydrophobic groups on the PLLA rough surfaces. Such a procedure was performed before in another biodegradable polyester, where electrospun 
poly ( $\varepsilon$-caprolactone) fabrics could exhibit a superhydrophobic character through a chemical vapour deposition methodology [21]. The morphology of the superhydrophobic surface changed considerably with respect to the previous methodssee figure 4 . The texture in this case is characterized by flower-like rods (figures $4(A),(B)$ and $(E)$ ) or papilla $(F)$. In figure $4(E)$ one can see that many leafy clusters are present in the cross section of the studied film. At low polymer concentrations leafy structures were obtained and at high polymer concentration spherulitic-like morphologies could be detected. The gradual transition from leafy structures to spherulitic structures with increasing polymer concentration is a commonly observed phenomenon and can be attributed to the fact that the nucleation and growth rates of the crystals increase at increasing polymer concentrations [24, 28]. The membranes immediately turned white after immersing the solution into the water coagulation bath. Conversely, the precipitation process was considerably slower upon immersion into ethanol. The precipitation in water was so quick that the membrane did not have enough time to fully crystallize during its formation. As a result, no leafy or spherulitic structures could be found at the surface-see figure $1(B)$. Figure $4(C)$ shows a magnified image of a typical single microcluster with a special flower-like structure composed of nano-fibrilar-like structures that are around $100 \mathrm{~nm}$ thick, 300-500 nm wide and several micrometres long. These micro-nano binary structures are thought to increase the surface roughness when compared with films exhibiting just roughness at the micro-level [25, 29]. From the image of the cross section of the prepared film, a typical single microcluster with a special flower-like structure composed of nano-fibrilarlike structures can also be found (figure $4(D)$ ), indicating that these special micro-nano binary structures exist in the whole film. In this hierarchical rough surface, the fraction of air in the pores in contact with the water drop is larger, and this fact accounts for the increase in water contact angle to values as large as $153.4^{\circ}[5,9,11]$. Note that in natural superhydrophobic surfaces, such as in the lotus leaves, a hierarchical structure is also observed combining micro- and nano-level roughness textures [9], and this fact has also been used to emphasize the extremely low wettability of such surfaces.

It is important to note that we could reach superhydrophobicity in PLLA films without the need to perform any chemical modification on the rough surface. Moreover, the rough PLLA surfaces were prepared through pure physical phase transformations, and therefore the chemical nature of the rough surface should be similar to the smooth film. Complementary Fourier-transformed infrared spectroscopy with attenuated total reflection experiments were performed, where we compared the transmittance spectra of the surface of the two films (results not shown): the same absorption bands could be found in each case and therefore we can conclude that the different wettabilities are just due to the different topography.

On a rough surface, two superhydrophobic states can be seen [27]: (i) Wenzel's state, where the drop follows perfectly the contour of the rough surface and the high contact angle of water originates from the increase of surface area, and (ii) Cassie's state, where a fraction of the drop contacts with air pockets existing between water and the rough solid surface. The fact that one could produce superhydrophobic surfaces (contact angle higher than $150^{\circ}$ ) from materials in which smooth films exhibit contact angles lower than $90^{\circ}$ is indicative that the pure Wenzel behaviour could not be consistent with the results, and that Cassie's state, alone or in combination with the Wenzel case, could explain the superhydrophobic nature of the obtained rough films.

\section{Conclusions}

Different approaches were tried to produce superhydrophobic PLLA surfaces, based on the precipitation of dioxane-based PLLA solutions into coagulation baths. Different variables were explored, namely the addition of a non-solvent in the PLLA solution, the addition of dioxane in the precipitation bath, the nature of the precipitation liquid and the PLLA concentration. A simple approach that could directly transform the moderately hydrophobic PLLA surface into a superhydrophobic surface without addition of low-surfaceenergy compounds was proposed by using a gelation-in-air procedure followed by precipitation in ethanol. The water contact angle of the top surface of the sponge-like porous film increased from $67^{\circ}$ to more than $150^{\circ}$. In the best formulations flower-like rods or papilla structures could be observed on the surface, combining roughness at a micro and nano level. The addition of a non-solvent was not beneficial for the increase of hydrophobicity.

\section{References}

[1] Lee Y, Park S-H, Kim K-B and Lee J-K 2007 Adv. Mater. 19 2330-5

[2] Sun T, Feng L, Gao X and Jiang L 2005 Acc. Chem. Res. 38 644-52

[3] Zhu Y, Zhang J, Zheng Y, Huang Z, Feng L and Jiang L 2006 Adv. Funct. Mater. 16568

[4] Zhao N, Weng L, Zhang X, Xie Q, Zhang X and Xu J 2006 Chem Phys Chem 7824

[5] Wang S, Feng L and Jiang L 2006 Adv. Mater. 18767

[6] Blossey R 2003 Nat. Mater. 2 301-6

[7] Barthlott W and Neinhuis C 1997 Planta 202 1-8

[8] Feng X and Jiang L 2006 Adv. Mater. 18 3063-78

[9] Feng L, Li S, Li Y, Li H, Zhang L, Zhai J, Song Y, Liu B, Jiang L and Zhu D 2002 Adv. Mater. 141857

[10] Wang Y, Liu Z, Han B, Sun Z, Zhang J and Sun D 2005 Adv. Funct. Mater. 15655

[11] Xie Q, Xu J, Feng L, Jiang L, Tang W, Luo X and Han C 2004 Adv. Mater 16 302-5

[12] van der Wal P and Steiner U 2007 Soft Matter 3 426-9

[13] Jiang L, Zhao Y and Zhai J 2004 Angew. Chem. Int. Ed. 434338

[14] Ma M and Hill R M 2006 Curr. Opin. Colloid Interf. Sci. 11 193-202

[15] Erbil H Y, Demirel A L, Avci Y and Mert O 2003 Science 299 1377-80

[16] Sodergard A and Stolt M 2002 Prog. Polym. Sci. 27 1123-63

[17] Kim H D, Bae E H, Kwon I C, Pal R R, Nam J D and Lee D S 2004 Biomaterials 252319 
[18] Kellomaki M, Niiranen H, Puumanen K, Ashammakhi N, Waris T and Tormala P 2000 Biomaterials 242495

[19] Khorasani M T and Mirzadeh H 2004 J. Appl. Polym. Sci. $912042-7$

[20] Sun T, Tan H, Han D, Fu Q and Jiang L 2005 Small 1 959-63

[21] Ma M, Mao Y, Gupta M, Gleason K K and Rutledge G C 2005 Macromolecules 38 9742-8

[22] Osawa S, Yabe M, Miyamura M and Mizuno K 2006 Polymer 47 3711-4

[23] Peng M, Li H, Wu L, Zheng Q, Chen Y and Gu W 2005 J. Appl. Polym. Sci. 98 1358-63
[24] van de Witte P, Esselbrugge H, Dijkstra P J, van den Berg J W A and Feijen J 1996 J. Polym. Sci. B $342569-78$

[25] Li X, Chen G, Ma Y, Feng L, Zhao H, Jiang L and Wang F 2006 Polymer 47 506-9

[26] Wang S and Jiang L 2007 Adv. Mater. 19 3423-4

[27] Li X-M, Reinhoudt D and Crego-Calama M 2007 Chem. Soc. Rev. 36 1350-68

[28] Lee I-C, Cheng L-P and Young T-H 2005 J. Biomed. Mater. Res. 76A 842-50

[29] Shirtcliffe N J, McHale G, Newton M I, Chabrol G and Perry C C 2004 Adv. Mater. 16 1929-32 